\section{Enfoques y postura crítica de la relación entre teología y neurociencias*}

\author{
Vicente Valenzuela Osorio**
}

RECIBIDO: 28-11-16. APROBADO: 19-04-17

Resumen: Se presentan los enfoques y crítica del encuentro de la teología y las modernas neurociencias, y se insiste en la necesidad de un diálogo auténtico entre la revelación en teología y lo que las neurociencias revelan en su propio discurso. El trabajo aporta una clave de interpretación, la revelación se da en la autonomía del mundo y afecta lo constitutivo de la teología, hasta el punto de exigir un giro en la pregunta por la fuente de la revelación: pasar de un modelo de trascendenciainmanencia, a un modelo de monismo relativo, donde la red neural humana se hace fuente constitutiva de la Palabra de Dios.

Palabras Clave: Relación entre neurociencia y teología; fuentes de la revelación; estatuto interno de la teología; monismo relativo; neuroteología.
Approaches and Critical Posture of the Relationship between Theology and Neurosciences

Aвsтract: The text presents approaches and critique of the encounter of Theology and Neurosciences, and the need for an authentic dialogue between revelation in Theology and what Neuroscience reveals in its own discourse. The work provides a key of interpretation, revelation occurs in the autonomy of the world and affects what is constitutive of Theology, to the point of demanding a turn in the question of the source of revelation: this means leaving behind a model of transcendence-immanence, and moving towards a model of relative monism where the human neural network becomes the source and constitution of the Word of God.

Keywords: Neuroscience and Theology; Source of Revelation; Internal Statute of Theology; Relative Monism; Neurotheology.

PARA CITAR ESTE ARTÍCULO:

Valenzuela Osorio, Vicente. "Enfoques y postura crítica de la relación entre teología y neurociencias". Theologica Xaveriana 185 (2018): 1-XX. https://doi.org/10.11144/ javeriana.tx68-185.epcrtn

* El presente artículo se configuró durante una pasantía de investigación en la Universidad Católica de Lovaina, en Bélgica, con la tutoría del profesor Benoît Bourgine. Además, aquí se busca evocar algunos resultados de la investigación de tesis doctoral, La carne como fuente primera y constitutiva de la teología. Testimonio teológico de la carne, articulado con una visión neurocientífica del mundo, llevada a cabo con el tutor Luis Orlando Jiménez.

** Doctor en Teología, Pontificia Universidad Javeriana, Bogotá. Docente de Teología sistemática en la Universidad de San Buenaventura, Bogotá. Orcid: 0000-0001-5022-1889. Correo electrónico: dominevico21@yahoo.com 


\section{Introducción}

El encuentro entre teología y las modernas neurociencias es de reciente data ${ }^{1}$. Este artículo no tiene por finalidad componer una historia del asunto; sin embargo, es oportuno decir que el trabajo investigativo en torno de los avances científicos del estudio del cerebro y del sistema nervioso, y su consiguiente visión antropológica, han provocado reacciones, algunas de las cuales se presentarán aquí como enfoques. Por otra parte, en el presente texto, las posturas críticas fueron aportando el dato de que la emergencia mundanal de la revelación no ha permeado lo suficiente el estatuto teológico ni el discurso neurocientífico.

Esto es así, siempre y cuando se acepte que la teología requiere dejarse configurar constantemente en su estatuto interno por su comprensión creciente de la revelación, a fin de tener algo con sentido para comunicar ante desafíos como los de las neurociencias ${ }^{2}$. En esta dirección se mueve la clave que surge a partir del análisis de los enfoques y la postura crítica hacia los mismos.

Los enfoques del encuentro han ido variando: desde un horizonte apologético que buscaba posicionar una religión natural en el ser humano (neuroreligión) o una base biológica de la pregunta por Dios (neuroteología), y luego, desde la indagación de la relación entre la antropología evolutiva, relacional de las neurociencias y las visiones de hombre en la teología (como en los apuntes antropológicos de Heinrich Rombach asumidos por Alexandre Ganoczy).

También se hace un esfuerzo más sistemático por reflexionar el lugar de la acción divina en las redes neurales, buscando una teoría ontológica de estructuras y niveles (Philip Clayton y seguidores) y sus respectivas críticas (Arthur Peacocke y Gregersen); se indaga acerca de la relación alma-cuerpo, se formula la pregunta por lo específico del ser humano (Benoît Bourgine, Bernard Feltz, Luis O. Jiménez), se

\footnotetext{
${ }^{1}$ Esa datación se obtiene al poner como punto de arranque los experimentos de estimulación magnética del cerebro, llevados a cabo, en los años 80, por Michael Persinger. Ellos permitieron a este hablar de una base neural de la creencia en Dios (Neuropsychological base of God beliefs); y de los primeros esfuerzos de las cuestionadas neuroreligión y neuroteología. Si se tiene en cuenta que las modernas neurociencias se constituyeron como saber científico entre 1950 y 1960, en los Estados Unidos, y que tenían por centro de interés el estudio interdiscipinar (de saberes médicos, tecnológicos, psicológicos y biológicos), del cerebro y del sistema nervioso, se aprecia que la reflexión sobre neurociencia y teología no lleva mucho camino recorrido.

2 "La théologie a-t-elle quelque chose à dire, qui serait digne d'être entendu, au sein du débat sur le spécifique humain? Et d'abord quelle théologie?" (Bourgine, "Darwinisme et évolution”, 11). "Qu’advientil par exemple lorsque le postulat biblique d'un statut spécifique de l'humain dans le cosmos, qui a si puissamment soutenu l'humanisme européen, est brutalement révoqué par des neuroscientifiques, extrapolant avec autant de légèreté que d'assurance les résultats de leur discipline à des affirmations de portée ontologique?" (ibíd., 12).
} 
aborda el asunto de los alucinógenos en la experiencia cristiana (varios autores) y otros temas teológicos, como cerebro, conversión y pecado (K. Bingaman).

\section{Métodología}

Para que el artículo no se presente como una información sobre el Status quaestionis, se realiza la pregunta por los enfoques de la relación entre teología y neurociencia, y como una clave interpretativa que va apareciendo (la revelación como una realidad que emerge desde la propia autonomía del mundo, dilucidada en el sentido evolutivo del sistema nervioso central, que repercute en la configuración de los discursos entre la visión neurocientífica y la teología), se puede ir formulando una crítica a cada enfoque con miras a que dicha clave sea asumida en términos de un diálogo auténtico.

Cada enfoque tiene su presentación y su respectiva crítica. Se dividirán en: (1) La neuroreligión y la neuroteología; (2) la antropología estructural de corte neurocientífico, comparada con la antropología bíblica, bajo el recurso de la analogía; (3) la ontología de niveles que permite hablar de la emergencia de la mente, conciencia y libertad y la acción de Dios; (4) alguna breve palabra sobre la relevancia de la investigación alma-cuerpo, mente-cerebro; aquí se indica la novedad de la pregunta por la especificidad humana; (5) el enfoque de investigación en teología, alucinógenos y neurociencia; y (6) otros acercamientos temáticos de la teología en relación al cerebro. Al final se dan las conclusiones. Sin embargo, cada postura crítica es conclusiva del análisis del enfoque ${ }^{3}$.

\section{Relación entre teología y neurociencia: enfoques y postura crítica}

\section{Neuroreligión y neuroteología}

\section{Enfoques}

Los primeros resultados de la investigación cerebral que utilizan los mecanismos de resonancia magnética (MRI) y de la imagen funcional obtenida por resonancia magnética (FRMI), producidos entre los años 80 y 90 del siglo pasado, fueron pretexto para

\footnotetext{
${ }^{3}$ En cuanto al propósito del presente artículo y su contenido temático, cabe hacer una aclaración: para obtener una visión conjunta de los enfoques, se recurrirá a un trato sintético de las diversas elaboraciones presentadas. En el trabajo de revisión y crítica de los enfoques, fueron apareciendo asuntos relevantes a la hora de pensar el camino que va abriendo la relación entre neurociencia y teología, y qué exigencias comienza a plantear este encuentro respecto del estatuto interno de la teología y de la revelación. No es un artículo sobre el estatuto de la teología, ni sobre la revelación, sino de reconocimiento del lugar en el que se sitúan los actuales debates y qué caminos se posibilitan para el enriquecimiento de la investigación teológica (ya sea porque los han abierto o porque adolecen de ellos).
} 
la aparición de una nueva reflexión acerca de la experiencia religiosa (neuroreligión) y de la experiencia mística y teologal (neuroteología). Las imágenes del encéfalo permitieron renovar el conocimiento que se tenía acerca de la relación entre cerebro y mente. Se comprendió que el cerebro es la base biológica de las emociones, el lenguaje, el pensamiento y otras dimensiones humanas. Con este criterio se optó por explorar realidades como las relacionadas con la mística y la contemplación ${ }^{4}$.

Desde la publicación de la novela de Aldous Juxley, Island (en la cual se articula el término neuroteología), ya se ofrecía una direccionalidad para esta región del saber: "¿Qué está sucediendo en el cerebro cuando se está teniendo una visión? ¿Y qué está sucediendo cuando se pasa de un estado mental premístico a uno auténticamente místico?"s Los teóricos de la neuroreligión y la neuroteología tienen como precedente a dicho autor, y también las indagaciones de Michael Persinger, a finales de los años 80 (estimulaba con electromagnetismo los lóbulos temporales para recrear el aura de experiencia extática $)^{6}$.

Otros pioneros de este saber fueron James Ashbrook y Carol Rausch Albright. Estos personajes tuvieron ideas profundas sobre la revelación y el cerebro que poco a poco fueron abandonadas en torno del cerebro como revelador de lo divino ${ }^{7}$. Después de ellos, otro estudioso, Laurence McKinney, basado en estudios de imágenes cerebrales, en casos de epilepsia, fue formulando el campo de la neuroteología. Esta consistía en determinar las áreas neurales que funcionaban cuando una persona estaba en actividad religiosa o en algún tipo de visión mística. Sus investigaciones apuntaban a establecer cuál es el lugar de Dios (God spot) en el cerebro ${ }^{8}$. En la misma línea de indagación se ubican autores como A. Newberg y Eugene D’Aquili. Ellos, en compañía de otros expertos, en el 2003, experimentaron con un grupo de monjas franciscanas en estado de meditación. Para ello usaron la técnica de tomografía computarizada del cerebro (Spect) ${ }^{\text {. }}$.

\footnotetext{
${ }^{4}$ Álvarez-Díaz, “¿Es posible la transdiciplinariedad entre neurociencias y religiones?”, 292.

${ }^{5}$ A. Huxley, Island (New York: Harper and Row, 1962), citado por Álvarez-Díaz, “¿Es posible la transdiciplinariedad entre neurociencias y religiones?", 292; Echarte, "Proyección y límites de la neuroteología en el pensamiento de Adous Huxley. La interdisciplinariedad a prueba en estudios existenciales", 44-45.

${ }^{6}$ Cooke y Elcoro, "Neurothelogy: Neuroscience of the Soul", 3.

7 "The brain is itself in some sense revelatory of the divine (Asbrook y Albright, 1997)" (Burns, "Christianity and the Cognitive Sciences", 308).

${ }^{8}$ Cooke y Elcoro, "Neurothelogy", 1-2. La obra de McKinney se titula Neurotheology: Virtual Religion in the 21st Century (1994).

${ }^{9}$ Cooke y Elcoro, "Neurothelogy", 4. En otro artículo se presenta un resumen del aporte de Newberg y los estudiosos de la neuroteología: "Neurotheology is the study of "how the mind/brain functions in terms of humankind's relation to God or ultimated reality' (Newberg, 1999). They offered a model for
} 


\section{Postura critica}

En el presente artículo solo se deja mencionado este primer enfoque y se da paso a otras alternativas. Esto, por tres razones:

1. La neuroteología secundaba la idea de una religiosidad natural en el ser humano, y se vio la religión como un aspecto necesario en la evolución humana ${ }^{10}$.

2. Los estudios que se mostraron como prueba de la religiosidad y de la necesidad de Dios solo probaban que, cuando una persona entraba en experiencia mística o de contemplación, se activaban ciertas zonas cerebrales, como la prefrontal; pero estas mismas zonas son las de actividad de atención y otras acciones humanas relacionadas con la conducta y la conciencia ${ }^{11}$; y un estudio científico, sin pretensiones religiosas, no podría pasar de esa descripción del fenómeno a fundamentar una experiencia religiosa natural.

3. Se conoce la zona de actividad neural pero no se sabe nada acerca de sus contenidos. En palabras del teólogo Hans Küng: "Las fascinantes imágenes del cerebro solo ofrecen por ahora información sobre dónde tienen lugar el pensar, el querer y el sentir, pero no -eso es lo que hemos constatado- sobre cómo acontecen y aún menos cuáles son sus contenidos"12. Este mismo teólogo ofrece la crítica a la neurociencia en los siguientes términos:

Por muy fecundo que pueda ser el estudio psicológico de los sentimientos, actos y experiencias religiosas, así como la comparación con fenómenos patológicos [...] poco aporta la "neuroteología" de ciertos apologetas evangelicales que desean desarrollar una prueba neurobiológica de la existencia de Dios apelando a que el ser humano necesita fe y la religión tanto como el comer y el beber. ${ }^{13}$

understanding how God is generated by the brain and yet at the same time is generative of material reality" (Burns, "Christianity and the Cognitive Sciences", 308). También se puede mencionar un estudio actual sobre la importancia de la meditación y su vinculación con los experimentos neurocientíficos. Este estudio es oportuno porque recopila el problema y abre una crítica: la experiencia interior sigue escapando al control de los experimentos de las neurociencias (Ricard, "Experiencia interior y neurociencias", 532-534).

${ }^{10} \mathrm{Kün}$, El principio de todas las cosas. Ciencia y religión, 186.

${ }^{11}$ Uno de los investigadores en neuroteología, Patrick McNamara, señala que el lóbulo prefrontal se activa en estado de meditación; pero también que es el área específica para comprender el comportamiento humano y algunas capacidades mentales (McNamara, "Frontal Lobes and the Evolution of Coperation and Religion”, 190-193). Esto, que puede ser argumento a favor de la religión en McNamara, también es un contra-argumento pues solo prueba asociaciones de actividades neurales en cierta zona identificada.

${ }^{12}$ Küng, El principio de todas las cosas, 186.

${ }^{13}$ W. James, The Varieties of Religious Experience. A Study in Human Nature (New York: 1902), citado por Küng, en El principio de todas las cosas, 186. Así mismo, "para una crítica, ver a F. W. Graf, "Brain me up! Gibt es einen neurobiologischen Gottesbeweis?", en C. Geyer (ed.), Hirnforschung und Willensfreiheit, 143-147 (ibíd.). 


\section{La similitud analógica entre antropología bíblica y antropología estructural}

\section{Enfoque}

El caso de los estudios de Alexandre Ganoczy es importante. Hacia 2008, él compara la antropología hebrea del Antiguo Testamento con las visiones antropológicas de las neurociencias, principalmente la estructural de Heinrich Rombach. A. Ganoczy cree que la Biblia supera el dualismo antropológico. Por ello anota desde el inicio que la Biblia no es platónica ni sigue un horizonte espiritualista; para ella, el hombre vive dentro de una pluralidad de momentos constitutivos; es un ser vivo en quien lo espiritual y carnal cohabitan en dichos momentos ${ }^{14}$.

Junto a las fuentes bíblicas, este autor busca reflexionar los aportes de la neurobiología y de las ciencias cognitivas. El sustrato filosófico es la filosofía de las estructuras en Spinoza y en Heinrich Rombach ${ }^{15}$. Acerca de la estructuración de lo metódico, Ganoczy habla de un "juego de una pluralidad convergente de métodos"16, que busca una adecuación, en el sentido aristotélico, entre cosa e inteligencia ${ }^{17}$.

El autor critica a Theilard de Chardin y a Karl Rahner ${ }^{18}$, y propone como comienzo del estado de la cuestión sobre la vinculación entre la antropología teológica

14 “La Bible n'est ni spiritualiste ni platonicienne. Elle ne dresse pas de frontières infranchissables entre le mortel et l'immortel. Elle a plutôt une vision de l'homme un dans la pluralité inextricable des 'moments' qui le constituent. Alors unité plurielle d'une animalité qui émerge en humanité? Structure 'holistique', comme disent des philosophes de la nature? Ou être si fortement 'in-carné' que personne ne devrait le disséquer -ne serait-ce qu'en pensé- en chair et en âme? Il faut percevoir l'homme comme vivant dans lequel tout ce qui est charnel est en même temps spirituel et tout ce qui est spirituel est en même temps charnel. Sans confusion ni séparation" (Ganoczy, Cristianisme et neurosciences. Pour une théologie de l'animal humain, 7). Un artículo actual trata sobre el trabajo de Ganoczy en la indagación por la mente-cerebro y cuerpo-alma (Jiménez Rodríguez, "Is it Reasonable to Upload a Non-reductive Christian Monism? Theology Confronted with the Mind-brain and Body-soul Problem").

${ }^{15}$ Ganoczy, Cristianisme et neurosciences, 8.

16 "Le jeu d'une pluralité convergente de méthodes" (ibíd., 9).

${ }^{17}$ Ibíd., 9-10.

${ }^{18}$ Por ejemplo, de Teilhard dirá -junto a Jacques Monod- que ese pensamiento está prendado de animismo que profesa una finalidad divinamente preestablecida y todo está haciéndose por fuerza de la divinidad y del Cristo (ibíd., 11). También asume la crítica a la teología de K. Rahner: el neurofisiólogo alemán Gerhard Roth dice de Rahner que, a pesar de haber trabajado con el paleontólogo Paul Overhage, no se dejó permear por la visión evolutiva del mundo. Su obra principal, Tratado fundamental sobre la fe, no tendría en cuenta la biología contemporánea ni las neurociencias: "Son grand ouvrage de synthèse Le traitéfondamental de la foi, ne tient aucun compte de la recherche biologique contemporaine et ignore les neurosciences. L'homme y apparaît d'emblée l'esprit mû par son 'expérience transcendantale', finalisé par son rapport ontologique au 'mystère absolu', inéluctablement ouvert à l'autorévélation de Dieu en Jésus Christ, mais freiné dans son élan par le péché d’origine. Gerhard Roth considère que la pensée rahnérienne 'se trouve entièrement hors de la pensée scientifique moderne'” (ibíd., 11). 
y la visión moderna de cerebro a W. Pannenberg. Dice de él que habló de la necesidad de una antropología teológica desde el recurso de la investigación científica ${ }^{19}$ : tras haber visto la compatibilidad entre hallazgos biológicos y la visión bíblica, dio el paso a hablar de imagen de Dios. Así habría preparado el camino al encuentro con la neurociencia ${ }^{20}$.

Para A. Ganoczy, dos autores se dedicaron por los años 90 a la relación entre teología y neurociencia: St. Niklaus Bosshard (explica cómo la naturaleza humana puede organizarse ella misma) y Caspar Söling (trata sobre el cerebro y cómo se vincula al problema del alma). También hace mención de J. C. Eccles y de su trabajo ${ }^{21}$. Acerca del estado actual de la teología, Ganoczy afirmaba que estaba retrasada en cuanto al dominio de la neurociencia ${ }^{22}$.

La antropología que Ganoczy ha tomado para su investigación es la estructural de Rombach: una estructura que tiene una complejidad creciente. La persona humana no se definiría por su ser substancial ni como compuesto porque, si se acepta la inmortalidad espiritual, el espíritu estaría siempre anhelando liberarse ${ }^{23}$. El modelo de la estructura es el sistema abierto (L. von Bertalanffly), en el que suceden energías hacia adentro y hacia afuera.

Esas energías se llaman "momentos". Y los momentos definen el proceso (ya que "momento sería un concepto cercano a movimiento), y configuran una ontología ${ }^{24}$. Junto a esto, Ganoczy implementa la analogía, no para hacer una analogia entis, sino

19 "Pannenberg pense que la réflexion sur la foi chrétienne ne peut que gagner à être attentive à la biologie moderne" (ibíd., 11). Ganoczy se refiere a Pannenberg, en la obra Antropologie in theologischer Perspektive (Gottingen: Vandenhpoeck et Ruprecht, 1983).

${ }^{20}$ Ganoczy, Cristianisme et neurosciences, 12.

${ }^{21}$ St. Niklaus Bosshard trataría: "Sur l'auto-organisation de la nature et de l'homme [erschafft die Welt sich selbst? Selbstorganisation von Natur und Mensch, aus naturwissenschaftlicher, philosophischer und theologischer Sicht (Fribourg-en-Br-Bâle-Vienne, Herder, 1985)]"; y Caspar Söling: "Sur le problème 'cerveau-âme' [Das Gehirm-Seele-Problem: neurobiologie und theologische Anthropologie, Paderborn, etc, 1995]" (ibíd., 13). Ganoczy también menciona a otros autores : "Le premier relate les acquis des neurosciences et en discute les tendances antagonistes, illustrées surtout par J. C. Eccles et R. W. Sperry, avant de les mettre en regard avec une anthropologie biblique. Le second commence par une introduction du neurobiologiste Herhard Roth y voit un 'essai hérö̈que' de raviver 'le dialogue totalement rompu entre la théologie chrétienne et la biologie moderne, en particulier la recherche sur le cerveau’" (ibíd., 13).

22 "À l'heure actuelle, j'ai le sentiment que la théologie est toujours en retard dans ce domaine" (ibíd.,13).

${ }^{23}$ Ibíd., 16.

24 "Rombach en effet n'entend pas par ce mot un laps de temps délimité, un 'maintenant' isolé, mais, selon une de ses acceptions allemandes, un événement ou processus décisif pour l'existence d'une chose ou d'une personne. Il lui confere ainsi une signification ontologique, c'est-à-dire relative à l'être. Tout ce qui existe est le résultat d'une multitude de 'moments', de mouvements, comme l'indique le mot latin momentum, forme contractée de movimientum, qui est à son origine” (ibíd., 17). 
de manera funcional, para entender mejor los procesos del cerebro del hombre como animal humano y para potenciar el cambio de antropología en la teología ${ }^{25}$.

Sobre su visión de cercanía entre la antropología bíblica y las evolutivas, Ganoczy resaltará la existencia de similitudes ${ }^{26}$. Porque tanto los orientales antiguos como los modernos han conocido la desaparición de especies y han podido tener la pregunta por su desaparición o transformación; pero también porque ya en los textos sapienciales se compara el comportamiento animal con el humano ${ }^{27}$. El autor establece, además, unas analogías con la noción de alianza y de animalidad en el hombre cuando la Biblia habla de carne ${ }^{28}$ y por ende, una relación comparativa (de traducción analógica), entre la visión bíblica del hombre y la de las neurociencias; pero resaltará que en el caso de la revelación hay algunas diferencias ${ }^{29}$.

\section{Postura critica}

1. A manera de juicio crítico, se podría decir: si bien el encuentro de visiones tendría lugar en la filosofía, el hecho de conceder la primacía a la analogía para establecer similitudes entre una antropología de la revelación bíblica y una estructural tomada de las neurociencias, oscurece el horizonte, ya que termina por establecer un préstamo analógico sin una razón crítica. Se trata de un trabajo de equiparación por sus similitudes, no por articulación racional.

2. Quizás el autor sigue viendo la imagen como portadora de la revelación; mas no logra ver que la imagen es en su capacidad manifestativa, el origen primero de la revelación. ¿Por qué? Porque ella es una operación dinámica de la carne, de todo el conectoma nervioso.

3. Sigue viendo la antropología como objeto y medio de lo divino, no como su fuente constitucional. Lo dice respecto de Jesús: "El hombre Jesús es considerado

\footnotetext{
${ }^{25}$ Ibíd., 22. "Les neurosciences invitent la théologie à changer son anthropologie dans ce sens. Elles attirent son attention sur la possibilité de discours comparatifs fondés sur la réalité expérimentable [...]. Le théologien pourrait, en partant de là, refonder son image de l'homme, qui ne serait pas forcément réduite à des rapprochements entre l'animal et l'homme, mais ouvrirait aussi de nombreuses possibilités inédites de parler du divin et de Dieu" (ibíd., 23).

26 "Le lecteur des textes relèvera sans peine certaines similitudes entre ce que disent les sages de la Bible, et des écologistes modernes" (ibíd., 47).

${ }^{27}$ Ibíd., 47-48.

${ }^{28}$ Ibíd., 55-56.

29 "Il apparait en effet que la tradition biblique se montre, dans ce qu' elle dit du comportement croyant, analogue à une anthropologie scientifique qui privilégie l'image concrète par rapport au concept abstrait" (ibíd., 93). Sin embargo, su comprensión de la revelación es central para marcar una crítica: "La révélation du divin se fait au moyen d'apparitions, de signes, de symboles, de métaphores, de paraboles, d'images parlantes qui donnent à penser" (ibíd., 93).
} 
como símbolo y el mediador de lo divino por excelencia" ${ }^{30}$. Acerca de ello cabe preguntar: ¿Es mediador o es la fuente en su carne misma de la divinidad y de su revelación?

4. Para Ganoczy, la relación antropológica entre Biblia y neurociencia se establece por la noción de corazón, Lebab, y por la situación neuronal de las emociones, sentimientos, voluntad y deseo; solo que en ellas ve un momento de ser creyente. Con eso le otorga importancia a la base emocional de la fe, pero le quita la fuerza al papel de las emociones como realizadoras de la revelación. Para él, las emociones son importantes en cuanto generan (uno de tantos momentos) un comportamiento creyente ${ }^{31}$.

Los puntos señalados se presentan como fundamentales en el encuentro entre visión neurocientífica y teología. Se trata de permitir este sin traducciones directas ni el establecimiento de similitudes analógicas, para respetar así sus distintos campos y métodos ${ }^{32}$. Al respecto se suele hablar, según la herencia de Jean Ladrière, de articulación reflexiva, en la cual se daría una "transgresión de semántica", no un préstamo sin mediación racional sistemática ${ }^{33}$.

Lo anterior condensaría el punto primero. En cuanto a los tres siguientes, para que una teología no tome las neurociencias como un utensilio (una razón instrumental del saber), es importante que la revelación hable desde su propia luz y que las visiones científicas del mundo puedan revelar a la revelación una luz propia y constitutiva de esta última. Así, quizás el aporte de las neurociencias sería determinante para aclarar la modalidad del ser de la revelación en la carne (Basar-Sarx).

La visión neurocientífica interpretada en el encuentro con la teología insistiría en la capacidad de las dimensiones humanas, vistas en un marco evolutivo, de ser constitutivas de Dios y de su Palabra (Dabar). El encuentro mencionado permitiría a la teología volver a pensar su estatuto interno y a considerar la manera en que la materia

\footnotetext{
30 "L’homme Jésus est considéré comme symbole et le médiateur du divin par excellence" (ibíd., 93).

${ }^{31}$ Ibíd., 154-155.

32 "An articulation of scientific worldviews in to Systematic Theology is possible through the mediation of Philosophy. In this articulation, Philosophy enables us to transform the semantics of scientific worldview to be used in Theology" (Jimenez Rodríguez, The Articulation between Natural Sciences and Systematic Theology. A Philosophical Mediation Based on the Contributions of Jean Ladrière and Xavier Zubiri, 38).

${ }_{33}$ Para el desarrollo de esta propuesta y de una "transgresión semántica", ver a Jimenez Rodríguez, The Articulation between Natural Sciences and Systematic Theology, 69-484. También en K. Rahner habría un esbozo de mediación filosófica (Rahner, "Natural Science and Reasonable Faith", 47-51). Existe la necesidad de mediar desde la interpretación, la existencia, el sentido, desde una razón reflexiva que se realice sobre lo contingente.
} 
está en dinámica de evolución, y en el caso del organismo humano en su propia autonomía, posibilita la experiencia que es llamada "de" Dios ${ }^{34}$ y así reafirma la kénosis divina ${ }^{35}$.

\section{Emergentismo y panenteísmo para un encuentro entre teología y neurociencias}

\section{Enfoque}

Existen otros enfoques con mucho impacto en los medios norteamericanos y europeos, como la propuesta más sistemática de Philip Clayton y algunas reacciones que suscita en otros autores.

Dicha propuesta puede caracterizarse de manera sintética en su capacidad de provocar una actitud conciliadora entre ciencia y teología, en un marco de saber en el cual los conceptos de la teología escolástica tengan cabida (causa eficiente, analogía, acto, acción divina, trascendencia, teísmo), pero también de ser un sistema maleable que permita la incorporación de nuevos conceptos tomados de la ciencia natural (evolución, emergencia, cerebro, qualias), conceptos de las teologías intérpretes de la Biblia (kénosis, panenteísmo, creación), y de algunas ontologías filosóficas (sistema, ontología, niveles, estructura, metafísica).

De esta forma, la propuesta de Clayton, de antemano, se mostraría como un esfuerzo de gran síntesis que permite la cohabitación de diversos relatos cuya finalidad es la actitud de diálogo, como se verá enseguida.

\footnotetext{
${ }^{34}$ Acerca de la pregunta por una antropología que crea a Dios versus una que percibe a Dios, se puede consultar a Fingelkurts y Fingelkurts, "Is Our Brain Hardwired to Produce God, or Is Our Brain Hardwired to Perceive God? A Systematic Review on the Role of the Brain in Mediating Religious Experience", 307-320.

${ }^{35}$ Esta fue la dirección asumida por la tesis doctoral en teología del autor del presente artículo, titulada "La carne vulnerable como fuente primera y constitutiva de la teología. El testimonio teológico de la carne, articulado con una visión neurocientífica evolutiva". Al reconocer dos asuntos (1) que en la relación ciencia y teología, alguna de las dos tendía a convertir la otra en un instrumento, y que para evitar esto era necesario reconocer el valor de la revelación en ambos casos; y (2) que en cuanto a lo antropológico, se hacía del ser humano una mediación de la expresión de Dios, pero que en el Evangelio de Juan y en las cartas auténticas de Pablo, como 1Corintios, esta visión antropológica quedaba superada, fue crucial dejar que la visión neurocientífica (mediante una articulación del lenguaje), posibilitara la reflexión acerca de la capacidad humana de ser fuente y no medio, y así informara la revelación bíblica; y que la revelación bíblica en Jesús aclarara el testimonio de dicha revelación que emerge desde la carne. En esta dinámica se vio que toda la teología, en su estatuto propio, quedaba reconfigurada. La idea de la realización del Dabar (Palabra-acontecimiento), en la persona de Jesús, fue asumida desde el aporte de von Balthasar, Gloria. Una estética teológica Vol. 7; la visión neurocientífica fue tomada de los siguientes estudios: Damasio, El error de Descartes. La razón de las emociones; ídem, Y el cerebro creó al hombre. ¿Cómo pudo el cerebro generar emociones, sentimientos, ideas y el yo?; y Llinás, El cerebro y el mito del yo. El papel de las neuronas en el pensamiento y el comportamiento humanos.
} 
Ph. Clayton comienza con el análisis de la relación entre ciencia y teología. Para él, las ciencias físicas parecen no ofrecer posibilidad, en sus marcos, de hablar de una acción divina en el mundo ${ }^{36}$. La comprensión de la ciencia física reside en que "el universo es un sistema físico cerrado" ${ }^{37}$; pero este inconveniente se debe a un equívoco en el uso del concepto de causa ${ }^{38}$. Si hay ambigüedad es porque hay abundancia de sentidos de causa que derivan en una pluralidad de niveles en la realidad: cada nivel con su propia forma de causalidad. Así, no sería lo mismo decir que Dios actúa (como causa) de igual manera en las realidades físicas que en la mente-libertad ${ }^{39}$.

Ahora bien, propuesto lo de los niveles de causalidad, ¿qué decir de la teología? Clayton propone -en la línea de Pannenberg- que una teología lo es cuando se pregunta por su propia verdad ${ }^{40}$. ¿Y de la relación de la teología con la ciencia? En ella, la teología "es una disciplina kenótica" ${ }^{4}$. No se trata de insertar la teoría de la evolución en una teología de la creación, sino de ver qué región de la teología de la creación permitiría el encuentro ${ }^{42}$. Así pues, esta región es la caracterización que le otorga la kénosis. Sin embargo, ¿cómo entender mente-conciencia en este horizonte kenótico? Para Clayton, el nivel de la explicación de los sistemas vivientes no elimina el campo de lo relativo al espíritu, sino unos y el otro conviven ${ }^{43}$. Para proponer una explicación, el autor recurrirá a una ontología de diversos niveles, donde cada nivel tiene su propio dinamismo. Cada uno de estos niveles está interrelacionado. Así, cabe pensar en los niveles de lo subatómico, lo atómico, lo molecular, lo celular, lo orgánico, mental, por solo mencionar algunos. Aquí es crucial la noción de emergencia: cada uno de estos niveles va posibilitando el otro, pero dentro de cada uno se da su propio reino de causalidades ${ }^{44}$.

Por su parte, la explicación de la emergencia de la mente puede ayudar a comprender el lugar de los postulados teístas, sin caer en reduccionismos. Por ejemplo, en

\footnotetext{
36 "Physical sciences, it appears, leaves no place for divine action" (Clayton, Adventures in the Spirit. Dog, World, Divine Action, 186). Esta es una de las obras más importantes del autor sobre el tema.

37 "The universe is a closed physical system" (ibíd., 186).

38 "Obey faces a certain threat of equivocation when one speaks of both God and physical objects as causes" (ibíd., 186).

${ }^{39}$ Ibíd., 197-198. En la libertad hay otra causalidad.

${ }^{40}$ Ibíd., 26-29.

41 "Is a kenotic discipline" (ibíd., 36).

42 "Rather, the question is what kind of doctrine of creation — and for that matter, doctrine of God — one might write in response to the fact of the ladder of emergence taken as a whole" (ibíd., 78).

43 “Althought the 'bottom-up' study of living systems does not require entelechies or a 'vial force', and althought the study of consciousness does not immediately presuppose a theory of Spirit or Geist, these fields do not need to be eliminativist" (ibíd., 75).
}

${ }^{44}$ Ibíd., 189-190. 
el paso de una física hacia una metafísica, y de allí a una teología, se puede hablar de ciertas propiedades emergentistas de la mente y del espíritu (en relación a la materia). Sin embargo, aún es insuficiente saber si todas las realidades espirituales son emergentes. Así pues, se llega a postular la posibilidad de una realidad personal espiritual de lo real, pues parece ser que las realidades del espíritu tienden hacia algo; y con ello también hacia una posible intencionalidad.

Así, Clayton da el paso de la metafísica a la teología. La emergencia postularía que Dios está comprometido con el ser humano y con otros mundos posibles. Eso sería en un encuentro constructivo con el teísmo tradicional y la metafísica. Quizás se pueda hablar allí de una emergencia débil (weak emergence, según Samuel Alexander); pero sería mejor entendido en un encuentro moderado con otras áreas del saber, bajo un modelo que les permita subsistir, esto es, en un emergentismo fuerte (strong emergence) que postule varios niveles de realidad ${ }^{45}$.

Clayton se inclina por una emergencia fuerte, por varias razones. Defiende los diversos niveles de complejidad y cómo también se obtienen niveles que posibilitan otros niveles ontológicos y epistemológicos. En esta complejidad creciente, cabe el diálogo entre ciencia, religión, teología, filosofía y metafísica, ya que no habría un solo nivel, ni siquiera una sola manera de las causas. Esto lleva a la necesidad de una nueva teoría sobre la causalidad ${ }^{46}$. En opinión de Javier Leach, cada nuevo nivel tiene sus propias leyes y estructura ${ }^{47}$, cada nivel es importante e irreductible. Por ejemplo, la ciencia amplía la visión y explicación del mundo, pero la física, en su progreso, no explica ni puede examinar la experiencia personal de los humanos y su valor. La conciencia sería uno de tantos niveles ${ }^{48}$.

J. Leach, al estudiar el emergentismo de Ph. Clayton, dirá que las tesis principales para entenderlo son las siguientes: un monismo ontológico que no se confunda con el monismo físico; una emergencia que no puede ser reducida a sistemas físicos de explicación; y una causalidad descendente que no se confunde con el causalismo físico. Este emergentismo es jerárquico y no dualista sino pluralista.

Ahora bien, en relación con la trascendencia, la aparición de la libertad marca nuevos niveles. Dios no necesitaría prediseñar el mundo, sino que esta libertad hablaría de la apertura del futuro del mundo. Esto lleva a otro problema, el de la

\footnotetext{
${ }^{45}$ Ibíd., 98-99.

${ }^{46}$ Ibíd. 98-99.

${ }^{47}$ Leach, "Emergence and Transcendence in Philip Clayton. His Moderate Ideas Place Him in an Ideal Position for Dialogue", 1109.

${ }^{48}$ Ibíd., 1110.
} 
acción divina (divine action). En un plano físico no es posible el milagro. Sin embargo, Dios actuaría en el nivel de los qualia (cuántico), en la indeterminación, y su acción sería real ${ }^{49}$.

$\mathrm{Ph}$. Clayton, en The Question of Human Freedom, dice que explicar la libertad en esta dinámica de niveles es fundamental ${ }^{50}$. Ella habría emergido a partir de otros niveles primitivos. En el trato de la conciencia-libertad ha habido muchos reduccionismos. Se han dado dos puntos extremos del asunto: la mirada naturalista que niega la libertad, y la mirada metafísica que la afirma. Sin embargo, la emergencia de la cultura es un buen ejemplo de su no reductibilidad. El autor buscará un diálogo entre ambas posturas: naturalista y metafísica ${ }^{51}$.

En cuanto a la pregunta teológica, esta consiste en averiguar si hay una realidad profunda a todo cuanto existe (Dios) que sea garante de la libertad. La ruta para establecer los límites será fenomenológica: apelar a la experiencia de autotrascendencia y a la búsqueda humana de autenticidad. Así recurrirá a la mediación filosófica de la cuestión. En su lenguaje, la pregunta por la autotrascendencia se torna en la pregunta por la acción humana, el ser humano que es actor (buman agency) ${ }^{52}$.

La emergencia de lo espontáneo y de la conducta específica de los organismos tiene una larga historia en los procesos de la evolución. En el ser humano eso está posibilitado por la complejidad de las redes del cerebro ${ }^{53}$. En la medida en que sus redes se van complejizando, en una emergencia, los niveles de libertad van ascendiendo ${ }^{54}$. Ahora bien, ¿cómo hablar de la libertad y de Dios en perspectiva de la emergencia?55

Para hablar de Dios se puede recurrir a dos caminos: el dualista platónico, o una visión ontológica de niveles de la realidad en la cual sea diferente el nivel natural del campo de la libertad ${ }^{5}$. En el caso de la pregunta, Dios y la libertad establecen una

${ }^{49}$ Ibíd., 1110-1112.

50 "The question of human freedom is one of these great questions. It is a classic philosophical aporia, to which some of the greatest minds in intellectual history have devoted sustained" (Clayton, In Quest of Freedom. The Emergence of Spirit in the Natural World, 11).

${ }^{51}$ Ibíd., 13-14.

${ }^{52}$ Ibíd., 15-17.

53 "With is some $10^{11}$ neurons and roughly $10^{14}$ neural connections" (ibíd., 133).

54 "It holds that the emergence of freedom is gradual and asymptotic; that there is an increasing freedom, or rather freedom-likeness, across the stages of biological and cultural evolution; and that one can therefore speak of agents who are, for all intents and purposes, free" (ibíd., 133).

${ }^{55}$ Ibíd., 135-136.

${ }^{56}$ Ibíd., 138. 
correlación ${ }^{57}$. El autor las ubica como una especial manera de la imago Dej ${ }^{58}$. Esta forma especial permite establecer analogías entre Dios y la libertad como entre las personas humanas y sus cuerpos ${ }^{59}$. Para Clayton, este sistema coherente sería el panenteísmo, donde la acción de Dios es posible -como se anotó antes- en el nivel de los qualias ${ }^{60}$.

\section{Postura critica}

Tras este recorrido, a manera de crítica de la propuesta de Ph. Clayton, se podría decir que el mayor problema que representa su sistema, en cuanto a la teología de la acción divina y al panenteísmo (cruciales en su visión teológica), es la apelación a la analogía. La postura de Clayton, en la que se nota el inconveniente de su sistema, es expuesta en un artículo de W. Brierlye, en The Oxford Handbook, editado por el mismo Clayton: la relación que tendría Dios con el cosmos es análoga a la que tiene una persona (en otros casos, la mente), con su propio cuerpo ${ }^{61}$.

La explicación de por qué es una dificultad aparece en la misma compilación de artículos. En el escrito de Thomas Owen se recuerda que Arthur Peacocke y Niels H. Gregersen hablaron ya de que esa analogía metafórica, si realzaba más la función de la mente sobre el cuerpo, tendía a separar la divinidad del mundo; pero que si acentuaba más la corporalidad de la mente, tendía a divinizar el mundo; o si, en el caso de que mantuviera un equilibro entre mente-cuerpo, se creaba un dualismo entre divinidad y mundo. Por ello, declaraban que no era una analogía pertinente para las modernas visiones científicas ${ }^{62}$.

\footnotetext{
57 "Correlations do not prove causes" (ibíd., 138-139).

58 "Correlations between specific notions of God and specific interpretations of human freedom are a particular form of imago Dei correlations" (ibíd., 139).

59 "In any given case the two sides are not identical, of course; yet in any coherent system they should at least be an analogous" (ibíd.,139).

${ }^{60}$ Ibíd. Leach (el estudioso de Clayton), en "Emergence and Transcendence in Philip Clayton", 1109, habla de lo real de esta acción.

61 "Clayton's theory of divine action rests on his panentheistic analogy: God's relation to the cosmos is like a person's relation to her or his body" (Brierlye, "The Potential of Panentheism for Dialogue between Science and Religion”, 642).

62 "The mind-body metaphor poses a problem for Panentheists in regard to transcendence and immanence, both human and divine. Panentheists usually clarify this metaphor by explaining that God is more than the world even as we are more than our bodies [...]. If they emphasize the essential character of embodiment, they tend to divinize the world. If they stress the idea what we are essentially selves or subjects, they imply a dualistic anthropology. As a result of these problems, Peacocke comes out against this metaphor, and Gregersen concludes that the soul-body metaphor for the God-world relation 'no longer commends itself' as an adequate contemporary order for the God-world relationship" (Thomas, "Problems in Panentheism", 657-658).
} 
Junto a esta crítica cabrían otras dos:

- $\quad$ Si bien se reconoce que las analogías de mente-cuerpo y Dios-mundo, no son oportunas, cabría preguntar si alguna analogía lo es. Con esto aparece el inmenso desafío de la teología de corte teísta y de modelos como el de $\mathrm{Ph}$. Cayton, que buscan conciliar una visión moderna con una visión clásica, de hablar de Dios y del mundo sin recurrir al establecimiento de analogías que tienden a volverse metafísicas. Lo que hay detrás de este asunto es lo siguiente: (1) ¿ Es posible la fundación de una metafísica que permita un panenteísmo conciliador del teísmo y del reino de las causas, a partir de una gramática como la de la mente-cuerpo? $\mathrm{Al}$ respecto existe un pequeño escrito de Max Black que plantea las dificultades del paso de la gramática a la metafísica del ser ${ }^{63}$. (2) Tomar radicalmente la noción bíblica de kénosis aplicada a la teología de la creación, ¿acaso implicaría elegir otras vías de lenguaje para hablar de Dios y de la posibilidad que tiene el mundo en su autonomía de dejar ser a Dios? ${ }^{64}$, (3) Esta recurrencia a un panenteísmo pensado a partir de la convivencia con un teísmo reinterpretado, ¿̨no es, en el fondo, lo que Raimon Panikkar denunciaba como el miedo de la teología al panteísmo y a sus implicaciones como un atreverse a pensar en otros límites superando las esquemas sustancialistas?: "El miedo a caer en el panteísmo permanece en muchas mentes en cuanto que no ha sido superado todavía el monoteísmo rígido de un Ser sustancia y esencialmente trascendente" ${ }^{65}$.(4) ¿Se justifica seguir pensando en términos de inmanencia y trascendencia, o más bien la situación exige cambiar de modelo para comprender la revelación?

- Ubicar la acción de Dios en el ámbito cuántico y crear un sistema ontológico de niveles, para permitir la cohabitación de visiones antiguas de mundo, como la teísta de causalidades. Este punto estaría preguntando por el grado de actitud y autenticidad en un cuestionamiento acerca de la verdad (como Clayton ha dicho siguiendo a Pannenberg), cuando este preguntar lo que busca es la cohabitación sintética de discursos de cara a un diálogo sin mayores cercos epistémicos (como lo exige la condición de buscar qué es la verdad).

\section{Mención de la indagación alma-cuerpo}

\section{Enfoque}

Existe una indagación sobre la relación cerebro-mente y cuerpo-alma. Aquí se dirán algunas breves palabras, ya que se trata de un campo amplio, que merece ser recogido

\section{${ }^{63}$ Black, Modelos y metáforas.}

${ }^{64}$ Un estudio sobre los efectos de la noción de kénosis en la teoría evolutiva, en Velez Caro, "Del Dios onmipotente a la 'humildad de Dios'. Una reflexión sobre la evolución en perspectiva kenótica”.

${ }^{65}$ Panikkar, La puerta estrecha del conocimiento. Sentidos, razón y fe, 110. 
en un trabajo dedicado especialmente a dicho tema. Se mencionarán aquí algunas ideas del autor italiano Giorgio Bonaccorso, pues este presenta un barrido metódico sobre el asunto en cuestión. Para él, la relación que establece entre la teología y la neurociencia gira en torno del problema antropológico, que aquí es asumido como cuerpo y alma.

La neurociencia, al ocuparse del cerebro, se centra en los sentimientos, las emociones y la acción. Por ello, Bonaccorso toca temas que en la teología tradicional se han atribuido al alma ${ }^{66}$. El problema central es la relación complejidad-unidad. Un asunto de la filosofía en la historia de Occidente ha sido relacionar unidad con simplicidad, y buscar explicarlo todo desde alguna ley o principio ${ }^{67}$.

Este autor seguirá hablando de alma, "pero no en el sentido de un principio simple e independiente de la realidad de la cual se debe principio"68. El alma ha sido tenida como principio de la vida y de la mente. Sin embargo, en estudios neurológicos, este principio ha sido atribuido a las funciones del organismo. Incluso en esto, numerosos autores han tenido problemas al relacionar la mente con los principios físicos. El error ha sido querer reducir el cuerpo a sus características físicas y por ello establecer un ruptura con las que no son físicas; pero si-por el contrario-se tiene que cuerpo es una realidad más compleja, se puede ver cómo emerge la mente ${ }^{69}$.

En el asunto puede haber algunas tendencias: un monismo reduccionista donde todo quiere ser explicado por los fenómenos físicos y no se reconoce la importancia de la experiencia en primera persona; o un dualismo que, para explicar la mente, sigue insistiendo en separar; o un monismo no reduccionista para el cual es importante la experiencia ${ }^{70}$.

Tampoco se puede reducir la experiencia en primera persona a una objetividad en tercera persona pues la realidad es compleja ${ }^{71}$. En esta complejidad pueden explicarse el cuerpo y el alma, pues el alma debe asumirse no como un añadido ni como realidad primordial, sino como realidad compleja del mismo cuerpo ${ }^{72}$. Esta complejidad ayuda

\footnotetext{
${ }^{66}$ Bonaccorso, "L’animma e il corpo alla luce delle neuroscienze", 61.

${ }^{67}$ Ibíd., 62.

68 "Ma non nel senso di un principio semplice e indipendente dall realtà di cui sarebbe principio" (ibíd., 62).

${ }^{69}$ Ibíd., 62-63.

${ }^{70}$ Ibíd., 64-65. En cuanto al monismo no reductivista, el autor Luis Orlando Jiménez insiste en la necesidad de no reducir las explicaciones a un materialismo, pues esto conlleva sus propias faltas lógicas internas (Jiménez Rodríguez, The Articulation between Natural Sciences and Systematic Theology, 372; e ídem, "Is it Reasonable to Upload a Non-reductive Christian Monism?".

${ }^{71}$ Bonaccorso, "L’animma e il corpo alla luce delle neuroscienze", 65.

72 "Intesa non come una realtà radicalmente diversa o addirittura opposta al corpo, ma come il più alto livello di complessità del corpo" (ibíd., 66).
} 
a entender la teología, pues la revelación se hace somática; no se trata de una infusión mental, sino que implica al hombre entero. En esto es enfático el cristianismo cuando habla de la encarnación y resurrección de la carne ${ }^{73}$.

\section{Postura critica}

A manera de pregunta sobre este enfoque, se puede cuestionar si, al saber que la revelación es somática (o la salvación, en el caso de la cita de Luis Orlando Jiménez, es dirigida a la carne y no a alma), es pertinente seguir hablando (como lenguaje reflexivo para la teología) sobre alma y cuerpo. Quizás la transformación de esta pregunta por el alma sea -en la indagación de los teólogos Benoît Bourgine, Bernard Feltz, y otros pensadores $^{74}$ (y la insistencia en un monismo no reduccionista, en Luis Orlando Jiménez $)^{75}$ - la búsqueda de lo específico del ser humano en relación con las otras criaturas, especialmente los animales. Tal cuestionar, de esta manera, vendría a representar un enfoque diferente a la sola pregunta por el alma como distintivo humano.

\section{Teología, neurociencias y alucinógenos}

\section{Enfoque}

Otra línea sobresaliente es el enfoque de teología, neurociencia y alucinógenos. Este asunto es pertinente al presente artículo pues en él subyacen varios postulados fundamentales en relación con la fundación de las grandes religiones y la experiencia mística. Por ejemplo, que los recolectores de alimentos que probaban las características de algunas plantas alucinógenas generaron experiencias chamánicas; o que la bebida del soma descrito en el Rig Veda (pudo de ser hongo amanita muscaria) está en la base de las religiones de la India; que los granos almacenados, por la humedad, adquirieron el hongo claviceps purpurea y estarían en el registro del comienzo de los misterios de Eleusys; como el peyote y el yagé para los indígenas centroamericanos, y del Amazonas, respectivamente ${ }^{76}$.

\footnotetext{
${ }^{73}$ Ibíd., 66. Esta idea es constante también en el trabajo de Jiménez Rodríguez, "Is it Reasonable to Upload a Non-reductive Christian Monism?", ya que, de acuerdo con San Ireneo de Lyon, insistirá en que la salvación se dirige a la carne y no al alma.

${ }^{74}$ Bourgine, Feltz, Laurent y Van den Bosch de Aguilar (dirs.). Darwinismes et spécificité de l'humain.

${ }^{75}$ Jiménez Rodríguez, "It is reasonable to upload a non-reductive Christian monism?"

${ }^{76}$ Nichols y Chemel, “The Neuropharmacology of Religious Experience: Hallucinogens and the Experience of the Divine", 3, 5-7. Por ejemplo, "Sven Liljeblad (1972), an American anthropologist, reported a belief commonly held by Native Americans that Peyote 'has come to the Indians to lead them to him as Christ came to the whites. As God reveals himself in Peyote, it becomes a sacrament whereby communion is established with him or with the spiritual world in general' (p. 103)" (ibíd., 7).
} 
El asunto también es pertinente porque en países como Estados Unidos la experiencia mística suscitada por drogas endógenas ha provocado discusiones en ámbitos eclesiales acerca de su legalización con fines místicos-terapéuticos ${ }^{77}$. En el caso de América Latina, las experiencias y visiones gestadas en el entorno del llamado "viaje" por el consumo de alucinógenos, provocan distintas visiones espirituales y religiosas de mundo. Estos serían los pretextos que hacen necesario incorporar aquí este enfoque como importante en el encuentro entre neurociencia y teología.

Los problemas que recrea el presente enfoque pueden presentarse en tres horizontes generales: en cuanto a la base de las religiones y experiencias místicas, en cuanto a la constitución de la narrativa espiritual y teológica, y, en relación a su función pública.

De lo primero cabe decir que existe el debate -como ya se ha mencionadode si los alucinógenos están en la base de las experiencias religiosas, y si también pueden ser el fundamento de experiencias teologales (esto es importante porque uno de los inconvenientes de los evangelizadores del nuevo continente, para aceptar las experiencias inducidas por el consumo de plantas sagradas, era que no veían allí lo crístico de la experiencia, ni la relación con la gracia ${ }^{78}$.

De lo segundo, se suscita la pregunta por el valor, significado y sentido de una experiencia inducida por el consumo de alucinógenos en comparación con una experiencia mística por contemplación o por gracia ${ }^{79}$. Este asunto es fundamental pues es la pregunta por lo constitutivo teológico de una experiencia que tiene la posibilidad de ser asumida por la teología y que, por ende, es capaz de tener su propia luz de la revelación, avalada por una comunidad creyente ${ }^{80}$.

Acerca de lo tercero, se hallan dos disputas: la función terapéutica de la experiencia mística provocada por alucinógenos y la institucionalización eclesial de dichas prácticas con fines terapéuticos-espirituales ${ }^{81}$.

\footnotetext{
${ }^{77} \mathrm{Al}$ respecto existen los siguientes trabajos: Hummel, "By Its Fruits? Mystical and Visionary States of Consciousness Occasioned by Entheogens"; Cole-Turner, "Entheogens, Mysticism, and Neuroscience”.

${ }^{78}$ Furst, "Alucinógenos y arquetipos", 19.

79 "Are drug occasioned mystical experiences neurologically the same as more traditional mystical states? Are there phenomenological and theological differences?" (Cole-Turner, "Entheogens, Mysticism, and Neuroscience", 642).
}

80 “'By their fruits shall ye know them?' It's good to learn that volunteers having even this limited experience had lasting benefits. But human history suggests that without a social vessel to hold the wine of revelation, it tends to dribble away. What conditions of community and practice best help people to hold on to what comes to them in those moments of revelation?" (Hummel, "By Its Fruits?", 685-886). El autor cita directamente a Huston Smith (Council on Spiritual Practices, http://csp.org/psilocybin/ psilopressbackground2008.pdf).

81 "From the perspectives afforded by James, I pose the following questions about the research at Johns Hopkins University described by Richards: (1) By what fruits might we assess the administration of this 


\section{Postura critica}

El problema entre neurociencias, teología y alucinógenos (endógenos) ha sido insuficientemente tratado. Cada uno de los tres aspectos generales señalados son fundamentales, pero el segundo (concerniente a la revelación) es vital para el debate y la aclaración de los otros dos. El problema que ha tenido este enfoque consiste en que ha sido tratado por estudiosos de la fenomenología y psicología de la religión (con horizontes etnológicos), por etnólogos propiamente ${ }^{82}$, o por teólogos pastores de comunidades cristianas ${ }^{83}$. En los dos primeros casos el asunto se torna descriptivo; en el tercero, se torna apologético de lo tradicionalmente entendido por experiencia cristiana, ya que el pastor está velando por el interés de la comunidad ${ }^{84}$.

Esto lleva a la necesidad de pensar el asunto al interior de la comunidad y de la vida pública, pero con una perspectiva crítica. ¿Por qué? Porque hay otras formas de comunidad engendradas por la autonomía del Espíritu Santo, sin mediación apostólica ${ }^{85}$. Este pudiera ser el caso de los grupos (personas) que buscan ampliar su horizonte espiritual y transformar su modo de ser en el mundo según una luz hallada en la experiencia inducida por alucinógenos y en el encuentro con la experiencia de la revelación. Quizás un elemento a considerar es el aporte de Raimon Panikkar, para quien el Cristo es más que el acontecimiento histórico de la Palestina de hace más de dos mil años ${ }^{86}$.

\section{Otros enfoques caracterizados por su reflexión sobre algún tema de la teología}

\section{Enfoques}

Hay otros tratos de la relación entre neurociencia y teología, pero se caracterizan, ante todo, por ser temáticos. Por ejemplo, preguntarse si en la conversión religiosa

drug? (2) And what practical bearings of entheogen use does this research attempt to assess? The second question shall be addressed first (Hummel, "By Its Fruits?", 687).

${ }^{82}$ Como en las obras de Furst, "Alucinógenos y arquetipos", o en el trabajo clásico de Schultes, Hoffmann y Rätsch, Plants of the Gods: Their Sacred, Healing, and Hallucinogenic Powers.

${ }^{83}$ Como en el caso de los artículos que la revista Zygon ha publicado (con intereses notoriamente apologéticos), de los ya citados: Cole-Turner, "Entheogens, Mysticism, and Neuroscience"; y Hummel, "By Its Fruits?".

84 "My conclusion in answer to these questions will be the following: At this point in time, I cannot conceive how religious practices of entheogen use could (should) even be considered (entertained) as viable practices within these Christian communities" (Hummel, "By Its Fruits?”, 691).

${ }^{85}$ Valenzuela Osorio, "Grace as the Form of the Church on the Basis of Saint Thomas Aquinas' Thought. An Ecclesiology for Context of Stigma, Stigmatization and Discrimination Involving HIV and AIDS", 102-103.

${ }^{86}$ Panikkar, La plenitud del hombre: una cristofania, 34-40. 
de personajes como San Pablo, San Agustín o John Wesley, la justificación en Cristo ha modificado inmediatamente a la persona humana, o esa justificación se ha vivido en un proceso de transformación cerebral ${ }^{87}$. En palabras de Alan Weissenbacher, la neurociencia no tiene nada que decirle a la teología de la conversión pues la justicia de Cristo no exige ningún proceso ${ }^{88}$.

Otro ejemplo puede ser el rastreado en algunos escritos que se preguntan por el status quaestionis de la relación entre ciencia y teología. Cabe resaltar dos estudios muy significativos del tema:

- La reflexión realizada en la escuela de Cracovia, en dos centros de investigación científica y filosófica, sobre la relación teología-ciencia, es heredera del tomismo, y en su caso, la neurociencia es tomada bajo sus categorías ${ }^{89}$.

- La reflexión realizada en las facultades católicas de teología del sur de Europa (Portugal, España e Italia), donde si bien se reconoce la importancia del aporte de las ciencias, la teología aún no las ha tomado lo suficientemente en serio como para reformar el curriculum a partir del encuentro con estas nuevas visiones, ni se ha dejado permear de la visión científica del mundo en lo constitutivo interno de la revelación ${ }^{90}$.

También existe una tendencia reflexiva que va siendo clásica. Se trata de la relación entre cerebro y ética, entre cerebro y teología moral. Si bien estos temas están vinculados a la discusión acerca de la libertad y la acción (como en los trabajos de Clayton, Rombach y Changeaux), cabe resaltar que se ha generado una discusión más amplia y crítica acerca de la función del discurso neurocientífico en el encuentro con la ética y la moral. Algunas características de dicha producción de saber son: (1) La necesidad de un discurso que no se reduzca a un dato de la biología y que logre incorporar la hermenéutica y la narratividad como transversales existenciales; (2) una

\footnotetext{
${ }^{87}$ En este artículo, en actitud defensiva, se opta por una modificación directa, donde no tienen que ver para nada los procesos neurales ni la palabra de la neurociencia (Weissenbacher, "The Neuroscience of Wesleyan Soteriology: The Dynamic of Both Instantaneous and Gradual Change").

88 "First, for doctrines of conversion that hold to the imputation of Christ's righteousness, neuroscience is irrelevant, because statements of instantaneous change are in terms of a relational status and not biological” (ibíd., 347).

${ }^{89}$ Brozek y Heller, "Science and Religion in the Kraków School”, 195-196. El artículo indaga en el Copernicus Center for Interdisciplinary Studies (1980) y en el Center for Interdisciplinary Studies (2008).

90 "Summarizing the results of this brief report about the relationships among science, religion, and Theology in Italy, Spain, and Portugal, a growing awareness and a steady progress can be observed, more evidently so in the last few years. However, the process has been very slow, delayed, and mostly isolated; indeed, some ambiguities still persist in this particular environment" (Oviedo y Garre, "The Interaction between Religion and Science in Catholic Southern Europe (Italy, Spain, Portugal)”, 190).
} 
actitud crítica para no pretender que la opción de intervenir y medicar el cerebro sea el camino superior para darle consistencia a la ética; (3) la moral exige una multidisciplinariedad para evitar la tentación de hacer del dato neurocientífico la autoridad moral por excelencia; y (4) las neurociencias no tienen la última palabra sobre la formación de la corporalidad humana ni el género. Esto significa que es importante no ceder al determinismo biológico, sino más bien entrar en diálogo con otras interpretaciones, por ejemplo, la que ve en el ser humano un ser falible en medio de un vasto mundo de opciones ${ }^{91}$.

El último ejemplo es (una contribución muy pertinente) acerca de neurociencias y pecado original. Kirk Bingaman escribe que la categoría "pecado original" es dañina para el ser humano y su transformación neuronal, y, por tanto, también para la realización de la comunicación de Dios. Dice que, antes de la revolución freudiana, el pecado original insistía en la culpabilidad, y que después de Freud, en la vergüenza ${ }^{92}$.

Sin embargo, esto significaría desde el punto de vista evolutivo que la capacidad del organismo de evitar el peligro para sobrevivir se introyectó bajo el influjo judeo-cristiano de pecado original y generó una negatividad al interior de la vivencia humana impidiendo así la conversión auténtica ${ }^{93}$. Por ello, Bingaman propone eliminar la noción de pecado original y devolverle el lugar positivo al sentimiento de protección: no incorporarlo sino hacer que vuelva a ser la capacidad de evitar el peligro exterior para proteger a los demás del peligro y del daño. Esto se lograría gracias a la plasticidad del cerebro y su capacidad de aprender ${ }^{94}$.

\section{Postura critica}

Los cuatro aspectos señalados recrean asuntos difíciles. Lastimosamente, no están articulados en una propuesta más sistemática, sino se presentan como visiones aisladas del problema.

Sobre la cuestión de la conversión inmediata o como proceso, cabe acotar que afirmar que los estudios biológicos del sistema nervioso no tienen ninguna palabra que decir es desconocer el acontecimiento central de la encarnación.

\footnotetext{
${ }^{91}$ El tema neurociencias y moral es trabajado en una publicación de la revista Concilium. Sobre el tema de una narratividad y hermenéutica, ver a Crichton, "La generosidad del filósofo", 539-546; acerca de evitar que las neurociencias se conviertan en un argumento superior, ver a Schleim, "Tras la búsqueda de la moral última”, 571-578; y a Hildt, “¿Cómo se relacionan el cerebro, la moral y la ética”, 581-586; el problema de género y cuerpo es tratado por Haker, "Identidad de género, cerebro y cuerpo", 599-604; y, sobre la importancia de no perder de vista la falibilidad humana, ver a Molinario, "Lo humano falible y las neurociencias", 611-618.

92 Bingaman, "Beyond Original Sin: A Paradigm Shift for the Age of Neuroscience”, 412-143.

${ }^{93}$ Ibíd., 413.

${ }^{94}$ Ibíd., 418-422.
} 
Acerca de los enfoques de la teología católica en algunos países y centros de investigación, es oportuno señalar que pareciera que la teología tomista-escolástica se ha revestido de un lenguaje científico moderno, pero en su estatuto interno sigue siendo impermeable, y por ello la relación que establece con la ciencia es exógena (caso de Cracovia); también, que la cuestión de la revelación sigue siendo endógena a la teología. Al pensarse hacia dentro, reconoce la importancia de la ciencia, pero no se deja hacer por su luz revelatoria. Así permanece inamovible en su epistemología, objeto y método (caso del sur de Europa).

Acerca de la ética y la moral, cabe resaltar la importancia del debate en torno del no reduccionismo de los discursos y el diálogo entre saberes. Cabe acotar que estos aportes sobre moral podrían estar vinculados a una discusión desde la teología fundamental, para que no parezcan un capítulo aparte de la teología, y hallen su clave de comprensión en una revisión de la epistemología teológica.

Sobre el tema del pecado original, es una postura que merece ser pensada e incorporada en la fundamentalidad teológica.

\section{Conclusiones}

Lo que aparece como problema para la relación teología-neurociencia, es: (1) Teología: la cuestión de cómo la revelación se encuentra en una autenticidad dialógica. No solo es la teología que elabora su discurso a partir de una formalidad (la luz de la revelación), sino que se deja constituir desde la visión del mundo y su propia manera de comunicar la revelación. En este punto se exige el paso de un modelo de revelación dualista (trascendencia-inmanencia) a un monismo relativo que, al recuperar la autonomía del mundo y de los procesos biológicos humanos, logre presentar la sensibilidad humana (red neural) como fuente constitutiva de la Palabra de Dios. (2) Neurociencia: la teología es una oportunidad para aportar en la reflexión al interior de la interdisciplinariedad de las neurociencias pues le invita a concebirse como discurso abierto a otras narrativas y a evitar así los reduccionismos metódicos (relativizar el monismo sistémico), de horizonte del saber y de su función en el mundo. La revelación en el discurso teológico también permite enriquecer la comprensión de lo que la neurociencia revela como luz propia.

Es importante tener la actitud de gestionar un diálogo auténtico, recurriendo a la mediación de la reflexión y la hermenéutica, para no crear sistemas sin un cerco epistémico capaz de realizar el discernimiento de los conceptos, enfoques y modelos. Es insuficiente hacer una síntesis del saber en la que se asuma una postura tibia frente a conceptos que ya resultan obsoletos para el encuentro entre teología y neurociencia (por ejemplo, en el caso de incorporar a ultranza el lenguaje metafísico, 
teísta o escolástico). Esto representa una invitación a la teología a dejarse configurar en su estatuto interno y en su lenguaje, para que así responda a los problemas de las visiones científicas del mundo, y sobre todo, a las búsquedas humanas quieren descubrir el sentido en un lenguaje de Dios.

No obstante, el encuentro entre neurociencias y teología requiere una mediación reflexiva entre ambos campos de lenguaje, de manera que se evite un intercambio directo a nivel semántico. Esto no impide que haya un encuentro auténtico pero, para llegar al mismo, se requiere la mediación conceptual. De esta forma, las neurociencias pueden aportar elementos vitales a la teología, que le permitan replantear su estatuto interno, por ejemplo, ayudar a comprender la manera como el sustrato biológico humano hace de la experiencia humana el lugar constitutivo de la revelación de Dios.

Es importante insistir en lo anterior, ya que después del rastreo de los enfoques se hace evidente que el encuentro sigue permeado por cierto temor a dejarse constituir por otro horizonte o visión. Por ello, o bien se recurre a sistemas analógicos, a sistemas que le concedan la razón a todo tipo de concepto (aunque pertenezca a una física del mundo antiguo), o sencillamente se hace del saber científico (visión neurocientífica) un utensilio para justificar posturas religiosas. Esto, cuando parece ser, por el contrario, que la dirección que emerge aquí invita a un pensar no utilitarista de las disciplinas, sino a una mutua constitución desde lo que cada una es en cuanto revelación. Así, el giro que se exige al discurso teológico es fundamental: la revelación tiene otra fuente, que es el ser humano en la autonomía de sus procesos evolutivos.

\section{Bibliografía}

Álvarez-Díaz, Jorge Alberto. “¿Es posible la transdiciplinariedad entre neurociencias y religiones?” Revista mexicana de neurociencias 15, 5 (2014): 291-296.

Bingaman, Kirk A. "Beyond Original Sin: A Paradigm Shift for the Age of Neuroscience”. Pastoral Psychol 61 (2012): 411-422.

Black, Max. Modelos y metáforas. Madrid: Tecnos, 1966.

Bonaccorso, Giorgio. "L'animma e il corpo alla luce delle neuroscienze". En Scienca e fede: un dialogo possibile? Evoluzionismo e teologia della creazione, editado por V. Danna y A. Piola, 61-68. Cantalupa (TO): Effatà Editrice, 2009.

Bourgine, Benoît. "Darwinisme et évolution". En Darwinismes et spécificité de l'humain, dirigido por B. Bourgine, B. Feltz, P-J., Laurent y Ph. van den Bosch de Aguilar, 11-14. Louvain-la-Neuve: Editions Academia, 2012. 
Bourgine, B.; Feltz, B.; Laurent, P-J.; y Ph. van den Bosch de Aguilar, Ph. (dirs.). Darwinismes et spécificité de l'humain. Louvain-la-Neuve: Editions Academia, 2012.

Brierlye, Michael. "The Potential of Panentheism for Dialogue between Science and Religion". En The Oxford Handbook of Religion and Science, editado por Ph. Clayton y Z. Simpson, 542-643. Oxford (U. K.): Oxford University Press, 2008.

Brozek, Bartosz y Michael Heller. "Science and Religion in the Kraków School”. Zygon Vol. 50, N.o 1 (2015): 194-208.

Burns, Charlene P. E. "Christianity and the Cognitive Sciences." En The Routledge Companion to Religion and Science, editado por James W. Haag, Gregory R. Peterson y Michael L. Spezio, 308-319. New York (NY): Routledge. 2014.

Clayton, Philip. Adventures in the Spirit. Dog, World, Divine Action. Minneapolis (MN): Fortress Press, 2008.

. In Quest of Freedom. The Emergence of Spirit in the Natural World. Göttingen: Vandenhoeck Ruprecht, 2009.

Clayton, Philip y Zachary Simpson (eds.). The Oxford Handbook of Religion and Science. Oxford (U. K.): Oxford University Press, 2008.

Cole-Turner, Ron. "Entheogens, Mysticism, and Neuroscience". Zygon Vol. 49, N. 3 (2014): 642-651.

Cooke, Paul y Mirari Elcoro. "Neurothelogy: Neuroscience of the Soul". Journal of Young Investigators 25, Issue 3 (2013): 1-6.

Crichton, Will. "La generosidad del filósofo y sus límites: Paul Ricoeur y la neurofilosofía." Revista internacional de teología Concilium 362 (2015): 535-546.

Damasio, Antonio. El error de Descartes. La razón de las emociones. Santiago de Chile: Andrés Bello, 1996.

. Y el cerebro creó al hombre. ¿Cómo pudo el cerebro generar emociones, sentimientos, ideas y el yo? Barcelona: Destino, 2010.

Echarte, Luis E. "Proyección y límites de la neuroteología en el pensamiento de Adous Huxley. La interdisciplinariedad a prueba en estudios existenciales". Persona y bioética 13, N. 1 (2009): 42-58.

Fingelkurts, Alexander A. y Andrew A. Fingelkurts. "Is Our Brain Hardwired to Produce God, or is Our Brain Hardwired to Perceive God? A Systematic Review on 
the Role of the Brain in Mediating Religious Experience". Cognitive Processing Vol. 10, N. ${ }^{\circ} 4$ (2009): 293-326.

Furst, Peter. "Alucinógenos y arquetipos". En Los alucinógenos y la cultura, por P. Furst, 50-55. México: Fondo de Cultura Económica, 1980.

Ganoczy, Alexandre. Cristianisme et neurosciences. Pour une théologie de l'animal humain. París: Éditions Odile Jacob, 2008.

Haker, Hille. "Identidad de género, cerebro y cuerpo". Revista internacional de teología Concilium 362 (2015): 589-604.

Hildt, Elisabeth. “¿Cómo se relacionan el cerebro, la moral y la ética?” Revista internacional de teología Concilium 362 (2015): 579-588.

$\mathrm{Hu}$, Huping y Maoxin Wu. "Scientific God: Michael Persinger \& the GOD Experiments." Scientific God Journal 3, Issue 10 (2012): 918-921.

Hummel, Leonard. "By Its Fruits? Mystical and Visionary States of Consciousness Occasioned by Entheogens". Zygon Vol 49, 3 (2014): 685-695.

Jiménez Rodríguez, Luis Orlando. "Is it Reasonable to Upload a Non-reductive Christian Monism?” Theology Confronted with the Mind-brain and Body-soul Problem”. Pensamiento Vol. 71, N. ${ }^{\circ} 269$ (2015): 1323-1345.

. The Articulation between Natural Sciences and Systematic Theology. A Philosophical Mediation Based on the Contributions of Jean Ladrière and Xavier Zubiri. Leuven-Paris-Bristol: Ephemerides Theologicae Lovanienses, 2015.

Küng, Hans. El principio de todas las cosas. Ciencia y religión. Madrid: Trotta, 2007.

Leach, Javier. "Emergence and Transcendence in Philip Clayton. His Moderate Ideas Place Him in an Ideal Position for Dialogue”. Pensamiento Vol. 64, N. 242 (2008): 1109-1113.

Llinás, Rodolfo R. El cerebro y el mito del yo. El papel de las neuronas en el pensamiento y el comportamiento humanos. Bogotá: Norma, 2003.

McKinney, Laurence. Neurotheology: Virtual Religion in the 21st Century. San Francisco (CA): American Institute for Mindfulness, 1994.

McNamara, Patrick. "Frontal Lobes and the Evolution of Cooperation and Religion". En Where God and Science Meet: How Brain and Evolutionary Studies Alter Our Understanding of Religion Vol. 2, 189-204. Westport (CT): Praeger Publishers, 2006. 
McNamara, Patrick (ed.) y otros. Where God and Science Meet: How Brain and Evolutionary Studies Alter Our Understanding of Religion. 3 vols. Westport (CT): Praeger Publishers, 2006.

Molinario, Joël. "Lo humano fallible y las neurociencias". Revista internacional de teología Concilium 362 (2015): 605-620.

Nichols, David E. y Benjamin R. Chemel. "The Neuropharmacology of Religious Experience: Hallucinogens and the Experience of the Divine”. En Where God and Science Meet. How Brain and Evolutionary Studies Alter Our Understanding of Religion. Volume 3. The Pshycology of Religion Experience, editado por Patrick McNamara, 1-33. Westport (CT): Praeger Publishers, 2006.

Oviedo, Lluís y Álvaro Garre. "The Interaction between Religion and Science in Catholic Southern Europe (Italy, Spain, Portugal)”. Zygon Vol. 50, N. ${ }^{1}$ (2015): 172-193.

Panikkar, Raimon. La plenitud del hombre: una cristofania. Madrid: Siruela, 2004. . La puerta estrecha del conocimiento. Sentidos, razón y fe. Barcelona: Herder, 2009.

Rahner, Karl. "Natural Science and Reasonable Faith”. Theological Investigations XXI. Science and Christian Faith, editado por Karl Rahner y M. Riley Hugh, 47-51. New York (NY): Crossroad, 1988. Disponible en: "CD compiled by the Centre for Culture, Technology and Values, Mary Inmmaculate College, Limerick, Ireland. Distributed by The Way, Campion Hall, Oxford, OXI 1QS": 16-55.

Ricard, Matthieu. "Experiencia interior y neurociencias". Revista internacional de teología Concilium 362 (2015): 521-534.

Schleim, Stephan. "Tras la búsqueda de la moral última. La investigación del cerebro en camino de la mano amiga para conseguir la autoridad moral". Revista internacional de teología Concilium 362 (2015): 561-578.

Schultes, Richard Evans; Albert Hofmann; y Christian Rätsch. Plants of the Gods: Their Sacred, Healing, and Hallucinogenic Powers. Rochester (VT): Healing Arts Press, 1998.

Society for Neuroscience. "About Neuroscience". SfN, https://www.sfn.org/about/ about-neuroscience (consultado el 20 de octubre de 2016).

. "History of SfN 1969-1995". SfN, https://www.sfn.org/about/history-of-sfn/ the-creation-of-neuroscience/introduction (consultado el 20 de octubre de 2016). 
Thomas, Owen C. "Problems in Panentheism." En The Oxford Handbook of Religion and Science, editado por Ph. Clayton y Z. Simpson, 652-664. Oxford (U. K.): Oxford University Press. 2008.

Valenzuela Osorio, Vicente. "Grace as the Form of the Church on the Basis of Saint Thomas Aquinas' Thought. An Ecclesiology for Context of Stigma, Stigmatization and Discrimination Involving HIV and AIDS”. En Theology and HIV \& AIDS in Latin America. A Collection of Essays by Master's Students from Four Latin American Academic Institutions, editado por Valburga S. Streck, 95-106. Sao Leopoldo: Oikos, 2013.

Vélez Caro, Olga Consuelo. "Del Dios omnipotente a la 'humildad de Dios'. Una reflexión sobre la evolución en perspectiva kenótica". Franciscanum LIV, N. ${ }^{\circ} 157$ (2012): 19-50.

Von Balthasar, Hans Urs. Gloria. Una estética teológica. Vol. 7. Madrid: Encuentro, 1989.

Weissenbacher, Alan C. "The Neuroscience of Wesleyan Soteriology: The Dynamic of Both Instantaneous and Gradual Change". Zygon Vol. 51, N. 2 (2016): 347-360. 